\section{Unexpected total recovery in a patient with post-anoxic complete alpha coma predicted by recording of cortical SEPs}

\section{Introduction}

Alpha-coma (AC) refers to EEG patterns that are predominantly in the alpha frequency band, recorded in unresponsive patients (Vignaendra et al., 1974; Iragui and McCutchen, 1983). AC has been described to occur after cardiorespiratory arrest (CRA), head trauma and brainstem lesions. Two types of post-anoxic AC have been identified: (1) the complete form which is characterized by a monotonous, continuous, frontally distributed and non-reactive EEG alpha activity; and (2) the incomplete form which is characterized by a non-monotonous, posteriorly accentuated and partially reactive EEG alpha rhythm. Complete AC is associated with poor prognosis, whereas the incomplete form of AC frequently leads to a good outcome and neurological recovery. Further, the complete form of AC is usually associated with abnormal or absent cortical somatosensory evoked potentials (SEPs) responses, while the incomplete form of AC demonstrates normal cortical SEPs components (Berkhoff et al., 2000).

\section{Case report}

A 27 year-old man entered a comatose state after CRA caused by Wolff-Parkinson-White syndrome. He had an out-of-hospital witnessed cardiac arrest and prompt cardiopulmonary resuscitation was initiated by an expert witness, until the paramedic team arrived. Advanced life support (ALS) resuscitation was started and sustained return of spontaneous circulation (ROSC) was obtained after 45 min (Fig. 1).

The patient was then transferred to our intensive care unit. On admission (day 0) his Glasgow Coma Scale (GCS) was three, with preservation of brainstem reflexes (Fig. 1). Urgent brain computed tomography was negative. Propofol sedation was started immediately after stable ROSC and maintained at the dose of $2.5 \mathrm{mg} / \mathrm{kg} / \mathrm{h}$ for $24 \mathrm{~h}$ (Fig. 1). After the first $24 \mathrm{~h}$, the propofol infusion rate was reduced to $0.5 \mathrm{mg} / \mathrm{kg} / \mathrm{h}$ and remifentanil therapy was added to maintain intubation and ventilation (Fig. 1). Daily monitoring of EEG was established in concomitance with the GCS assessment during the wake-up tests that were obtained $4 \mathrm{~h}$ after propofol and remifentanil discontinuation. During wake-up tests the patient remained comatose, with no spontaneous eye opening and no verbal or motor responses (GCS of three). On day 1 of admission, the EEG showed the presence of an alpha background activity that was non-reactive to verbal and painful stimuli, evident in the frontal areas, and that became more stable and monotonous on the third day after admission. On day 7, the EEG documented an alpha background activity that was monotonous, non-reactive, frontally distributed, but unstable with intermixed rare diffuse delta waves. On day 10 , the EEG showed a stable, non-reactive and frontally distributed alpha background activity (Fig. 2A). The EEG spectral analysis underlined the pathological predominant alpha frequency that was more evident in the frontal regions compared to the occipital areas (Fig. 2B and C). SEPs were measured after the stimulation of the bilateral median nerves (Fig. 1). Notably, the latency of the cortical N20 components of SEPs was normal on the right side, but delayed on the left side (Fig. 2D1 and D2). At this time, brain magnetic resonance imaging was unremarkable. The patient remained comatose (GCS of three) and the progression of his condition was complicated by the development of myoglobinuria and acute renal failure that required continuous renal replacement therapy. Other treatments started at this time included amiodarone and broad-spectrum antibiotics to treat the aspiration pneumonia that had developed.

Unexpectedly, on day 21 , the patient became responsive to painful stimuli and his GCS improved to six (Eye-1, Verbal-1, Motor-4) (Fig. 1). The EEG showed an unstable and frontally prevalent alpha background activity, with the presence of diffuse theta waves after painful stimuli. On day 26 the patient regained consciousness and was able to perform simple tasks. At this time the GCS was 14 (Eye-4, Verbal-4, Motor-6) (Fig. 1). The EEG, performed on the alert patient, documented an alpha background activity that was regular and reactive, predominant in the occipital areas, but unstable at times replaced by beta and theta rhythms more evident in the bilateral frontal-central-temporal regions (Fig. 3A). Indeed, the EEG spectral analysis showed the alpha dominating frequency more evident in the posterior regions compared to the frontal areas (Fig. 3B and C). During the following two days, the patient achieved a complete neurological recovery. Upon neurological examination, he appeared conscious, alert and able to speak, and did not exhibit sensory-motor deficits (GCS 15). Moreover, the patient regained normal renal function and the placement of an implantable cardioverter defibrillator was performed. When the patient was able to walk, he was discharged from the hospital and sent to a specialized neurological unit to start a period of recommended rehabilitation.

\section{Discussion}

Although several reports of incomplete AC with good prognosis are present in literature (Berkhoff et al., 2000), there are very few described cases of complete AC with favorable outcome (Rothstein, 2004). At the beginning of the past decade, Berkhoff et al. emphasized the importance of distinguishing, in terms of prognostic value, the AC in the complete and incomplete form. These authors showed that the EEG reactivity, and the unstable and posteriorly accentuated alpha background activity characterize the incomplete form of AC. This form seems to be associated with a less 


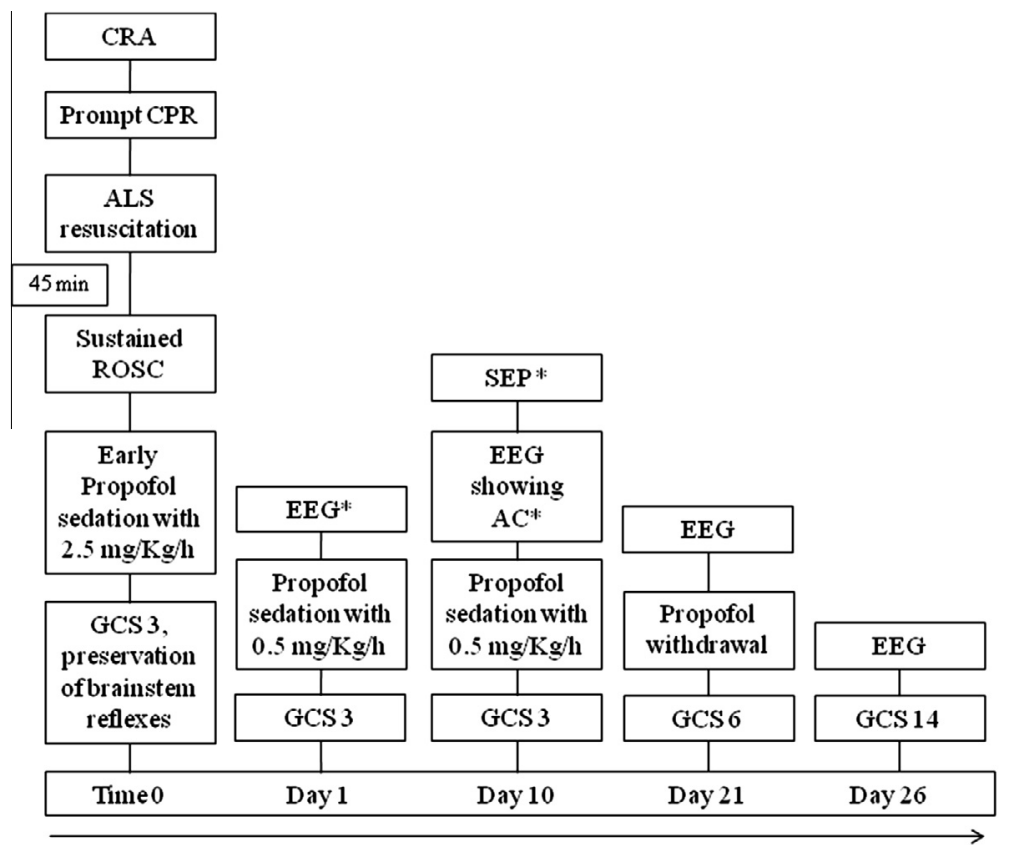

Fig. 1. Flow chart that shows the temporal evolution of clinical signs and the timing of the neurophysiological procedures. *Propofol infusion was discontinued $4 \mathrm{~h}$ before each neurophysiological assessment.

A

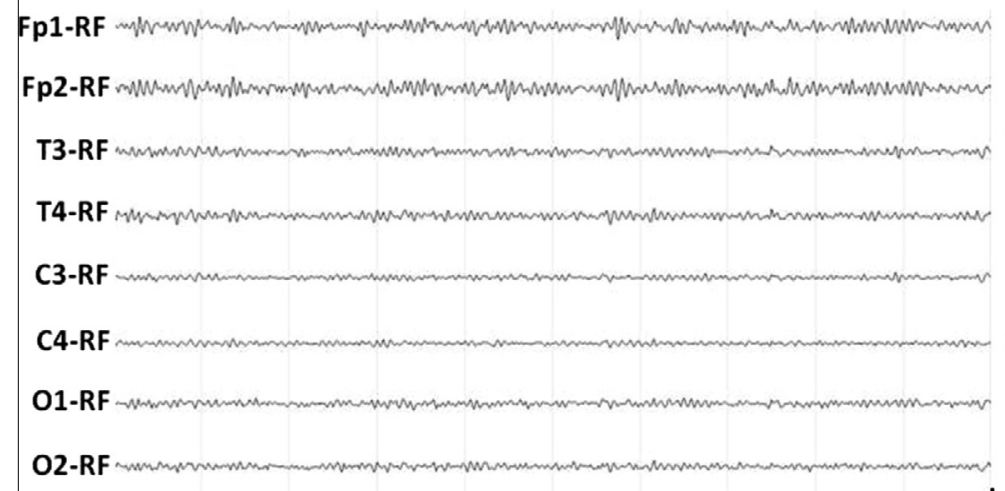

Day 10

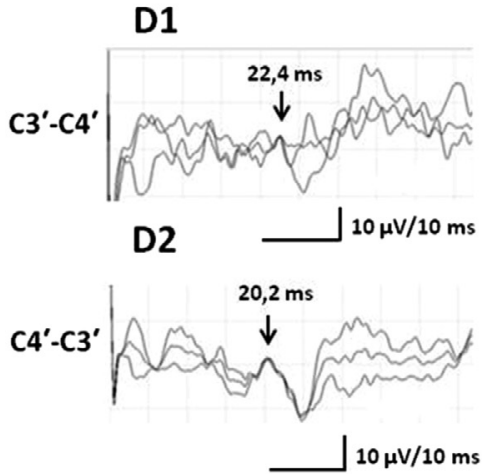

$7 \mu \mathrm{V} / 1 \mathrm{sec}$
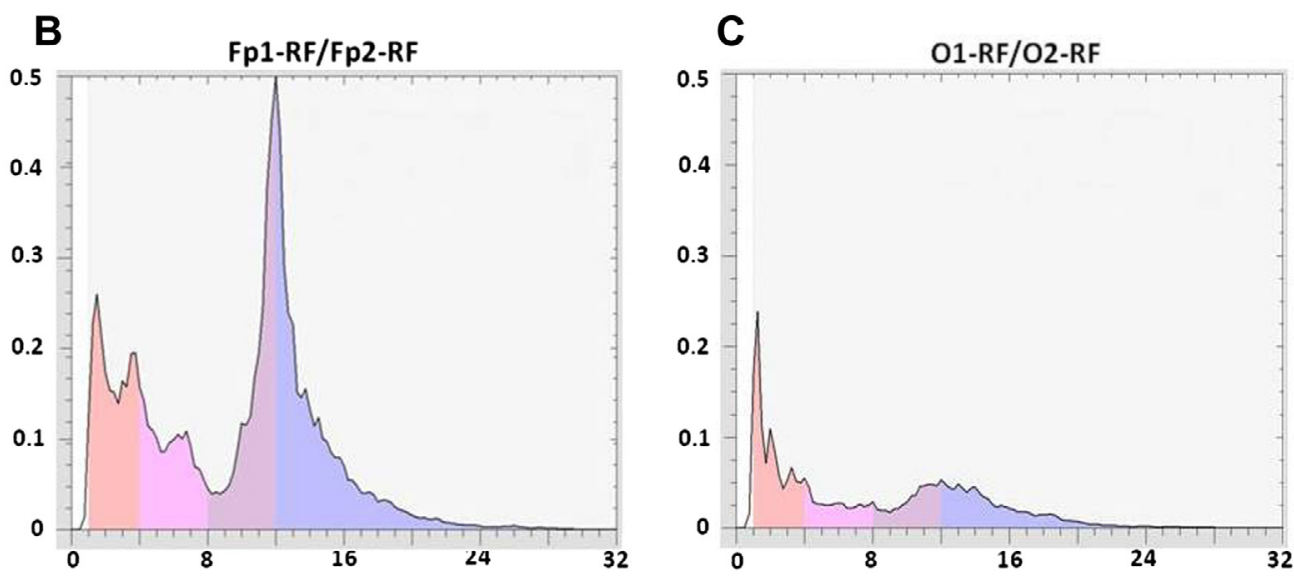

Fig. 2. Day 10 (A) EEG performed on comatose and unresponsive patient showing a stable alpha background activity, frontally prevalent and non-reactive. (B and C) EEG spectral analysis of 147 four-second epochs showing alpha dominating frequency on the frontal regions (aggregated FFT data across Fp1/Fp2) (B) compared to the occipital areas (aggregated FFT data across 01/O2) (C). (D1 and D2) SEPs measured on the comatose patient after stimulation of the bilateral median nerve. Electrodes were placed on $\mathrm{C}^{\prime}$ ( $2 \mathrm{~cm}$ posterior to $\mathrm{C} 3$ ) and C4' (2 cm posterior to C4) of the international 10-20 system. SEPs recording showed the presence of cortical N20 responses, with mild prolonged latency on the left side (D1) and normal latency on the right side (D2). RF, biauricular reference electrode. 
A

Day 26

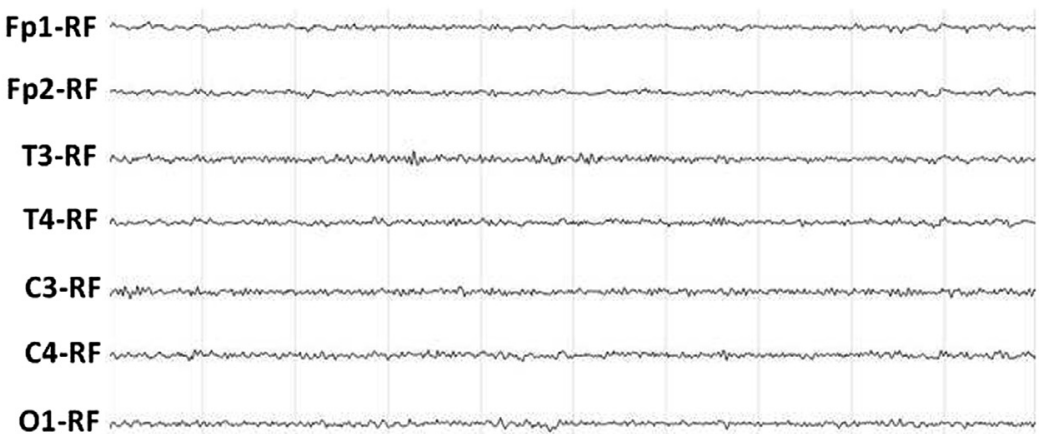

O2-RF $7 \mu \mathrm{V} / 1 \mathrm{sec}$

B

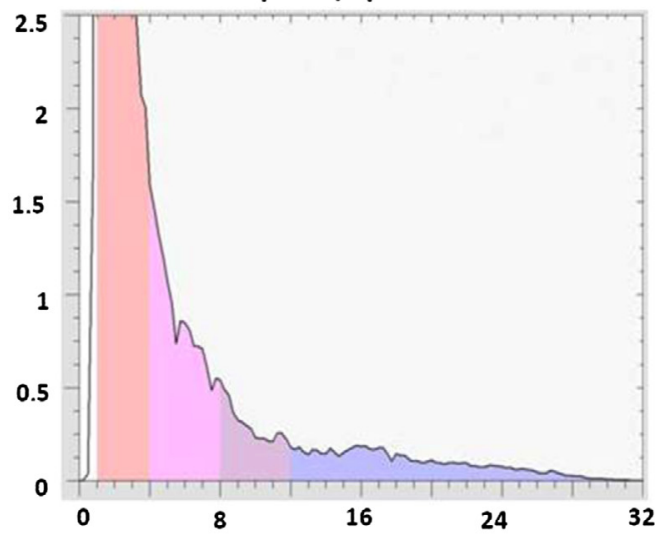

C

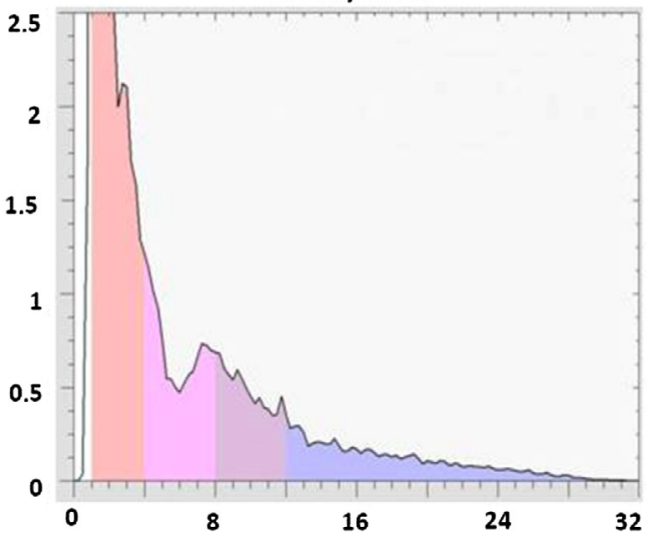

Fig. 3. Day 26 (A) EEG performed on the alert patient showing a regular, reactive and symmetric alpha background activity, intermixed by some theta and beta waves more evident on the bilateral frontal-central-temporal regions. (B and C) EEG spectral analysis of 137 four-second epochs showing the alpha dominating frequency on the occipital regions (aggregated FFT data across 01/O2) (C) with respect to the frontal areas (aggregated FFT data across Fp1/Fp2) (B). RF, biauricular reference electrode.

severe degree of brain injury, and a higher possibility to regain consciousness. In fact, the incomplete form of AC has a more favorable prognosis. Notably, the recording of cortical SEPs has been strongly associated with the incomplete form of AC (Berkhoff et al., 2000), whereas the absence of the N20 cortical responses on SEPs, associated with the complete form of AC, indicates an unfavorable prognosis.

It has been previously described that EEG rhythms, and in particular the alpha activity, respond promptly to ischemia, while the latency between an ischemic event and the drop of SEPs amplitude could take up to several minutes (Wess et al., 2010). It has been hypothesized that N20 cortical responses of SEPs reflect an intact mode to receive input stimuli, while EEG rhythms mainly reflect the brain's ability to send output commands (Van Putten, 2012). In this view, cortical neurons and their synapses seem to be more sensitive to hypoxia when sending output signals, as recorded by EEG, compared to receiving inputs, as recorded by cortical SEPs (Van Putten, 2012).

In our case, the CRA caused a severe injury of the cortical neurons as evident on the EEG, which showed the progressive appearance of a complete, monotonous, non-reactive and frontally prevalent alpha background activity. Contextually, the patient presented a stable neurological status with GCS of three. On this basis, we predicted an unfavorable outcome. However, during the complete AC status, we recorded the presence of the cortical N20 components of SEPs.
Surprisingly, two weeks later, the EEG abruptly changed and reactivity to painful stimuli was recorded. A few days later, the patient regained consciousness, and gradually achieved a total recovery.

The outcome of our patient is probably due to the efficient CPR promptly performed after the CRA and the contextual systemic vasoconstriction response, which warranted a cerebral perfusion with hypoxic levels unable to provoke irreversible cortical damage. In fact, the hypoxic injury to cortical structures temporarily altered neurons that generate EEG rhythms, but not neurons involved in the generation of cortical N20 SEPs components. Hence, the patient regained a total recovery when cerebral structures restored their functions after the transient hypoxic injury.

It is reported that complete AC is invariably associated with brain death, persistent vegetative state and poor outcome; on the other hand, the presence of the cortical components of SEPs may indicate a favorable prognosis (Berkhoff et al., 2000; Carter and Butt, 2005).

In the case of our patient with complete AC, we recorded the N20 cortical responses of SEPs, and he unexpectedly achieved a total recovery. Therefore, recording of cortical SEPs seems to be a more valuable test for establishing the prognosis of comatose patients after CRA than the EEG documentation of complete AC.

In conclusion, our case demonstrates that complete AC may be associated with good outcome if cortical N20 responses of SEPs are recorded. 


\section{References}

Berkhoff M, Donati F, Bassetti C. Postanoxic alpha (theta) coma: a reappraisal of its prognostic significance. Clin Neurophysiol 2000;111:297-304.

Carter BG, Butt W. Are somatosensory evoked potentials the best predictor of outcome after severe brain injury? A systematic review. Intensive Care Med 2005;31:765-75.

Iragui VJ, McCutchen CB. Physiologic and prognostic significance of "alpha coma". J Neurol Neurosurg Psychiatry 1983;46:632-8

Rothstein TL. Recovery from near death following cerebral anoxia: a case report demonstrating superiority of median somatosensory evoked potentials over EEG in predicting a favorable outcome after cardiopulmonary resuscitation. Adv Exp Med Biol 2004;550:189-96.

van Putten MJ. The N20 in post-anoxic coma: are you listening? Clin Neurophysiol 2012;123:1460-4

Vignaendra V, Wilkus RJ, Copass MK, Chatrian GE. Electroencephalographic rhythms of alpha frequency in comatose patients after cardiopulmonary arrest. Neurology 1974;24:582-8.

Wess C, Sarnthein J, Krayenbühl N, Scholz M, Kunze E, Meixensberger J. Spectral iEEG markers precede SSEP events during surgery for subarachnoid hemorrhage. Clin Neurophysiol 2010;121:2172-6.

C. Liguori Neurophysiopathology Unit, Department of Systems Medicine, University Hospital of "Tor Vergata", Viale Oxford 81, 00133 Rome, Italy Tel.: +39 0620902107; fax: +390620902106. E-mail address: dott.claudioliguori@yahoo.it
M. Albanese

A. Romigi

F. Izzi

Neurophysiopathology Unit, Department of Systems Medicine, University Hospital of "Tor Vergata", Rome, Italy

S. Natoli

F. Leonardis

C. Leonardis

Department of Clinical Sciences and Translational Medicine Intensive Care Unit, University Hospital of "Tor Vergata", Rome, Italy

L. Bianchi

Department of Civil Engineering and Computer Science Engineering, University of "Tor Vergata", Rome, Italy

M.G. Marciani

F. Placidi

Neurology Unit, Department of Systems Medicine, University Hospital of "Tor Vergata", Rome, Italy IRCCS Santa Lucia Foundation, Rome, Italy

Available online 5 July 2013 